\title{
Around and around: The expectations hypothesis
}

\author{
Mark Fisher \\ Research and Statistics \\ Board of Governors of the Federal Reserve System \\ and \\ Christian Gilles* \\ Monetary Affairs \\ Board of Governors of the Federal Reserve System
}

May 1996

*Mail Stop 74, Federal Reserve Board, Washington, DC 20551; phone: (202) 452-2567; email: cgilles@frb.gov. The views expressed herein are the authors' and do not necessarily reflect those of the Board of Governors or the Federal Reserve System. We thank Greg Duffee for extensive comments and many useful discussions. 


\begin{abstract}
We show how to construct arbitrage-free models of the term structure of interest rates in which various expectations hypotheses can hold. McCulloch (1993) provided a Gaussian non-Markovian example of the unbiased expectations hypothesis (U-EH), thereby contradicting the assertion by Cox, Ingersoll, and Ross (CIR, 1981) that only the so-called local expectations hypothesis could hold. We generalize that example in three ways: $(i)$ We characterize the $\mathrm{U}-\mathrm{EH}$ in terms of forward rates; $(i i)$ we extend this characterization to a class of expectations hypotheses that includes all of those considered by CIR; and (iii) we construct stationary Markovian and non-Gaussian economies. The building block is a maturity-dependent vector that travels around a circle at a constant speed as maturity increases.
\end{abstract}




\section{Around and around: The expectations hypothesis}

\section{Introduction}

In one form or another, the expectations hypothesis has played a central role in the analysis of the term structure of interest rates. Perhaps the most common form of the expectations hypothesis is the so-called unbiased expectations hypothesis (U$\mathrm{EH})$ that asserts that forward rates equal the conditional expectations of future spot rates, but other forms exist as well. Cox, Ingersoll, and Ross (1981) (CIR) characterized a number of mutually incompatible forms of the expectations hypothesis, including, besides the $\mathrm{U}-\mathrm{EH}$, the local expectations hypothesis ( $\mathrm{L}-\mathrm{EH})$, under which the expected rate of return on all zero-coupon bonds (on all assets, in fact) equals the short-term risk-free rate. Of the various expectations hypotheses they considered, they claimed that only the L-EH was consistent with general equilibrium in continuous-time models. ${ }^{1}$

McCulloch (1993) provided a counter-example to CIR's claim. His example is in the spirit of Heath, Jarrow, and Morton (1992) (HJM), in the sense that it does not admit a representation in terms of a finite number of Markovian state variables. Indeed, McCulloch suggests that CIR's claim may be true within the framework of an economy with a finite number of Markovian state variables. As we show, it is not.

There is a weak version of the U-EH according to which forward rates are biased predictors of future spot rates, but the bias, or term premium, is a constant that only depends on the forecast horizon. In this weak version, a regression of future spot rates on current forward rates has a slope coefficient equal to unity, but the intercept is unrestricted. ${ }^{2}$ To construct an arbitrage-free model of the yield curve in which the weak U-EH holds, it is sufficient to let all volatilities be constant - the Gaussian case. ${ }^{3}$ By contrast, our objective is (in part) to construct models of the strong version of the U-EH.

In this paper, we first generalize McCulloch's example by putting the U-EH explicitly into the HJM framework, focusing on absence-of-arbitrage conditions rather than building from a general equilibrium model. ${ }^{4}$ We then extend the analysis to a class of expectations hypotheses, parametrized by a scalar $q$, that includes as special cases those considered by CIR. Finally, we show how to construct Markovian examples: We start with two-state-variable stationary Markov economies that are Gaussianthat is, volatilities are nonrandom. Then, we show how to construct non-Gaussian

\footnotetext{
${ }^{1}$ They extended their claim to discrete-time models where interest rates are continuously compounded.

${ }^{2}$ See, for example, Campbell and Shiller (1991), who tested, and rejected, a weak version of the $\mathrm{U}-\mathrm{EH}$.

${ }^{3}$ See Campbell (1986) for a one-factor, general-equilibrium example.

${ }^{4}$ In Appendix A we show how to interpret the results from a general equilibrium perspective.
} 
two- and three-state-variable models. The two-state-variable Gaussian model and the three-state-variable non-Gaussian model (but not the two-state-variable nonGaussian model) are members of exponential-affine class of term structure models characterized by Duffie and Kan (1993).

The building block for the construction of any of our examples is a vector-valued function $\phi(t, T)$, where $t$ is the current time and $T$ is the maturity date. As long as, for any fixed $t, \phi(t, T)$ lies on a sphere, the expectations hypothesis holds. If, in addition, the path of $\phi(t, t+\tau)$ describes a circle on the sphere and goes around that circle at a constant speed as $\tau$ increases, then the model is Markovian.

\section{The $\mathrm{U}-\mathrm{EH}$ in an $\mathrm{HJM}$ setting}

In this section, which serves to introduce the analysis, we generalize McCulloch's example by characterizing the $\mathrm{U}-\mathrm{EH}$ in terms of the HJM absence-of-arbitrage restriction.

Let $P(t, T)$ denote the price at time $t$ of a default-free zero-coupon bond that pays one unit of account at time $T$. Assume that, at any time $t, P(t, T)$ is a differentiable function of $T$, and define the instantaneous forward rate as

$$
f(t, T):=-\frac{\partial}{\partial T} \log [P(t, T)]
$$

which, of course, implies

$$
\log [P(t, T)]=-\int_{s=t}^{T} f(t, s) d s,
$$

and define the short rate at time $t$, which is assumed to exist, as

$$
r(t):=\lim _{T \rightarrow t} f(t, T)
$$

Let $E_{t}[\cdot]$ be the conditional expectation operator. ${ }^{5}$ Following CIR, we define the $\mathrm{U}-\mathrm{EH}$ as follows: Forward rates are the conditional expectation of future spot rates; i.e,

$$
f(t, T)=E_{t}[r(T)]
$$

In the HJM approach to modeling the term structure, the primitives are $(i)$ an initial yield curve $\{f(0, t) \mid t>0\}$, (ii) the process for the market price of risk

\footnotetext{
${ }^{5}$ The concept of equivalent martingale measures hinders rather than facilitates the analysis of expectations hypotheses. As a result, we make no use of it and all expectations are taken with respect to the physical measure.
} 
$\lambda(t),{ }^{6}$ and $(i i i)$ the volatility of forward rates. We restrict attention to economies in which forward rates are diffusions driven by a $d$-dimensional vector $W(t)$ of standard Brownian motions. Let the process for forward rates be ${ }^{7}$

$$
d f(t, T)=\mu_{f}(t, T) d t+\sigma_{f}(t, T)^{\top} d W(t),
$$

where $\sigma_{f}(t, T)$ is a $d$-dimensional vector of forward-rate volatilities. Note that the market price of risk, $\lambda(t)$, is also a $d$-dimensional vector, and that it could be a random process, as could $\mu_{f}(t, T)$ and $\sigma_{f}(t, T)$.

It is now straightforward to derive the relationship between expected future short rates and current forward rates. Since $r(T)=f(T, T)=f(t, T)+\int_{s=t}^{T} d f(s, T)$, we can write

$$
E_{t}[r(T)]=f(t, T)+\int_{s=t}^{T} E_{t}[d f(s, T)]=f(t, T)+\int_{s=t}^{T} E_{t}\left[\mu_{f}(s, T)\right] d s .
$$

Define the forward rate premium as follows:

$$
\psi(t, T):=f(t, T)-E_{t}[r(T)]=\int_{s=t}^{T} E_{t}\left[-\mu_{f}(s, T)\right] d s .
$$

From (4), it is evident that if forward rates are unbiased predictors of future spot rates, then forward rates are martingales: $\mu_{f}(t, T) \equiv 0$. If, in addition, an ergodic yield curve exists, then it is flat. An upward sloping ergodic yield curve would require an expected decrease in forward rates on average.

It might seem easy, then, to construct examples of the U-EH: simply choose processes for forward rates $(3)$ with $\mu_{f}(t, T) \equiv 0$. The problem is that doing so arbitrarily might introduce arbitrage opportunities. The HJM absence-of-arbitrage condition specifies the drift of forward rates $\mathrm{as}^{8}$

$$
\mu_{f}(t, T)=\sigma_{f}(t, T)^{\top}\left(\lambda(t)+\int_{s=t}^{T} \sigma_{f}(t, s) d s\right)
$$

for all $0 \leq t \leq T$. Equation (5) shows that we must be able to write forward rate drifts in terms of their volatilities and the market price of risk. It follows directly that the HJM characterization of the $\mathrm{U}-\mathrm{EH}$ is

$$
\sigma_{f}(t, T)^{\top}\left(\lambda(t)+\int_{s=t}^{T} \sigma_{f}(t, s) d s\right)=0 .
$$

\footnotetext{
${ }^{6}$ See Appendix A for a discussion of the market price of risk.

${ }^{7}$ We use $(\cdot)^{\top}$ to denote transposes of vectors and matrices.

${ }^{8}$ We derive this expression in Appendix A. This was first shown by HJM; see also Duffie (1996), p. 151 or Hull (1993), p. 398-401. The form of our restriction differs from the form that Duffie and Hull give because in their presentations the drift is risk adjusted, while here $\mu_{f}(t, T)$ is not.
} 
The modeling challenge, then, is to find $\left\{\lambda(t), \sigma_{f}(t, T)\right\}$ pairs that satisfy (6).

To meet this challenge, it is convenient to define

$$
\phi(t, T):=\lambda(t)+\int_{s=t}^{T} \sigma_{f}(t, s) d s .
$$

Note that $\phi^{\prime}(t, T)=\sigma_{f}(t, T)$, where we define $F^{\prime}(t, T):=\frac{\partial}{\partial T} F(t, T)$ for any function $F(\cdot, \cdot)$. Using $(7)$, we can write $(6)$ as

$$
\phi^{\prime}(t, T)^{\top} \phi(t, T)=0 .
$$

Any function $\phi(t, T)$ that satisfies (8) has constant length: $\|\phi(t, T)\|=\|\phi(t, t)\|$. Two comments are in order. First, note that when $d=1$, this condition can be satisfied only if $\phi(t, T)$ is a constant function of its second argument, in which case $\sigma_{f}(t, T) \equiv 0$, which means there is no uncertainty. Thus in order for (8) to hold when interest rates are stochastic, there must be at least two Brownians. Second, note that (8) does not restrict how $\phi(t, T)$ behaves as a function of its first argument: In particular, $\phi(t, T)$ can be a stochastic process.

We can restate the key relationship between $\phi(t, T)$ and the U-EH as follows:

If $\phi(t, T)$ is a rotation of $\phi(t, t)$, then the $\mathrm{U}-\mathrm{EH}$ is satisfied in an arbitragefree way.

We now have a simple recipe for constructing arbitrage-free models of the U-EH: (i) choose $\phi(t, T)$ such that $\phi(t, t)$ is some random process and $\phi(t, T)$, for $T>t$, is a rotation of $\phi(t, t)$; $(i i)$ define $\lambda(t)=\phi(t, t)$, and $(i i i)$ define $\sigma_{f}(t, T)=\phi^{\prime}(t, T)$.

McCulloch (1993) constructed an economy in which the U-EH holds. ${ }^{9}$ In McCulloch's example there are two sources of risk, so that $d=2$. He chose $\mathrm{e}^{10}$

$$
\phi(t, t+\tau)=a\left(\sqrt{2 e^{-\tau}-e^{-2 \tau}}, 1-e^{-\tau}\right)^{\top},
$$

where $a=\eta \sqrt{g_{0}}$ in his notation. Note that $\|\phi(t, t+\tau)\|=\|\phi(t, t)\|=a$. Clearly, as $\tau$ increases, $\phi(t, t+\tau)$ turns continuously from $\phi(t, t)=(a, 0)^{\top}$ to $\phi(t, t+\infty)=(0, a)^{\top}$ going a quarter of the way around the circle over the infinite horizon. Finally note that McCulloch's example is Gaussian since $\phi(t, T)$ is deterministic.

\section{A class of expectations hypotheses}

In this section, we generalize the results from the previous section to encompass an entire class of expectations hypotheses. For this purpose, we will need to refer to the

\footnotetext{
${ }^{9}$ Frachot and Lesne (1994) noted that such an example could be constructed easily by exploiting equation (6).

${ }^{10}$ We reversed the order of McCulloch's Brownian motions for comparison with what follows.
} 
process for zero-coupon bonds: ${ }^{11}$

$$
\frac{d P(t, T)}{P(t, T)}=\mu_{P}(t, T) d t+\sigma_{P}(t, T)^{\top} d W(t)
$$

From (2), note the following relation between the volatility of bond prices and that of forward rates

$$
\sigma_{P}(t, T)=-\int_{s=t}^{T} \sigma_{f}(t, s) d s
$$

CIR characterized four versions of the expectations hypotheses: the U-EH, the L-EH, the Yield-to-Maturity Hypothesis (YTM-EH), and the Return-to-Maturity Hypothesis (RTM-EH). They showed that the U-EH and the YTM-EH are identical in continuous time. ${ }^{12}$ CIR went on to show that-after imposing absence-of-arbitrage conditions - the three independent expectations hypotheses could be characterized in the following way:

$$
\sigma_{P}(t, T)^{\top} \lambda(t)=\frac{q}{2}\left\|\sigma_{P}(t, T)\right\|^{2}
$$

where

$$
q= \begin{cases}0 & \text { under L-EH, } \\ 1 & \text { under YTM/U-EH, and } \\ 2 & \text { under RTM-EH. }\end{cases}
$$

Equation (11) provides an equilibrium (or absence-of-arbitrage) characterization of the expectations hypotheses. Moreover, it shows that the three hypotheses are mutually inconsistent unless $\sigma_{P}(t, T) \equiv 0$. Although CIR only considered $q \in\{0,1,2\}$, we allow $q$ to be an arbitrary real number, and we refer to (11) as the $q$-expectations hypothesis $(q-\mathrm{EH})$. CIR referred to (11) in making their claim that only the L-EH could hold in a continuous-time general equilibrium model. Clearly, the L-EH has a special status, since $q=0$ implies $\lambda(t)$ is orthogonal to $\sigma_{P}(t, T)$ but imposes no other restriction; with $\lambda(t) \equiv 0$, for example, the $\mathrm{L}-\mathrm{EH}$ is always satisfied and $\sigma_{P}(t, T)$ is unrestricted. For any other value of $q$, by contrast, if $\lambda(t)$ is orthogonal to $\sigma_{P}(t, T)$, then $\sigma_{P}(t, T)=0$.

We can recast (11) in terms of forward rates by differentiating both sides with

\footnotetext{
${ }^{11}$ In such a process, we call $\mu_{P}$ the proportional drift of $P$; the drift of $P$ is $\mu_{P} \times P$, of course.

${ }^{12} \mathrm{CIR}$ showed that the two hypotheses are identical in discrete time too if interest rates are continuously compounded.
} 
respect to $T$, using (10), and rearranging:

$$
\sigma_{f}(t, T)^{\top}\left(\lambda(t)+q \int_{s=t}^{T} \sigma_{f}(t, s) d s\right)=0 .
$$

We see that (6) is a special case of (12) with $q=1$. It is convenient to generalize the definition of $\phi(t, T)$ : Define $\phi(t, T)$ implicitly by

$$
\lambda(t)=q \phi(t, t)
$$

and

$$
\sigma_{f}(t, T)=\phi^{\prime}(t, T)
$$

so that $q \phi(t, T)=\lambda(t)+q \int_{s=t}^{T} \sigma_{f}(t, s) d s$.

Using (13-14), we can write (12) as

$$
q \phi^{\prime}(t, T)^{\top} \phi(t, T)=0
$$

Equation (15) is satisfied automatically if $q=0$. If $q \neq 0,(15)$ reduces to (8), in which case the comments that follow (8) apply here to the generalized definition of $\phi(t, T)$.

The recipe for constructing arbitrage-free models of the $q-\mathrm{EH}$ is this: $(i)$ choose $\phi(t, T)$ such that $\phi(t, t)$ is some random process and $\phi(t, T)$, for $T>t$, is a rotation of $\phi(t, t) ;(i i)$ define $\lambda(t)=q \phi(t, t)$, and (iii) define $\sigma_{f}(t, T)=\phi^{\prime}(t, T)$. For example, with McCulloch's $\phi(t, T)$, we could choose $\lambda(t)=q \phi(t, t)$ for any $q .{ }^{13}$

Finally, note that we can restate the $q-\mathrm{EH}$ in terms of either forward rate drifts or term premia. Using (13-14), we can rewrite the no-arbitrage condition (5) as

$$
\mu_{f}(t, T)=\phi^{\prime}(t, T)^{\top}((q-1) \phi(t, t)+\phi(t, T)) .
$$

For $q \neq 0,(15)$ implies

$$
\mu_{f}(t, T)=(q-1) \phi^{\prime}(t, T)^{\top} \phi(t, t),
$$

which in turn, in view of (4), holds if and only if

$$
\psi(t, T)=(1-q) \int_{s=t}^{T} E_{t}\left[\phi^{\prime}(s, T)^{\top} \phi(s, s)\right] d s .
$$

In what follows, we assume for convenience that (8), (16), and (17) hold even when $q=0$.

\footnotetext{
${ }^{13}$ The underlying general equilibrium would look different of course. See Appendix A.
} 


\section{Markovian models}

McCulloch (1993) established decisively that the unbiased expectations hypothesis is consistent with general equilibrium. But he left open the possibility that expectations hypotheses may be inconsistent with general equilibrium in the finite-state Markovian world analyzed by CIR. We settle this issue by exhibiting two- and three-state variable stationary Markov economies. The construction allows for the volatility of bond prices to be stochastic. The trick is to make $\phi(t, T)$ proceed around a circle at a constant pace, producing an infinite number of cycles.

In all of the examples we develop below, the processes for the short rate and its drift share the following structure:

$$
d r(t)=x(t) d t+\omega \zeta(t) d W_{2}(t)
$$

and

$$
d x(t)=y(t) d t-\omega^{2} \zeta(t) d W_{1}(t) .
$$

Note that the diffusion for $r(t)$ depends only on $W_{2}(t)$, while the diffusion for its drift $x(t)$ depends only on $W_{1}(t)$, which is orthogonal to $W_{1}(t)$. As we will see, this seemingly capricious ordering of Brownians follows from our canonical representation for $\lambda(t)$.

To facilitate the analysis of the examples, we define a pair of functions that we will use repeatedly and for which $(q, \omega, \mu, z)$ is a vector of fixed parameters:

and

$$
Y(r, \zeta):=\omega^{2}\left(\mu-r+(q-1)\left(z^{2}-\zeta^{2}\right)\right)
$$

$$
F_{q}(r, x, \tau):=\mu+(r-\mu) \cos [\omega \tau]+x \frac{\sin [\omega \tau]}{\omega}+(q-1) z^{2}(1-\cos [\omega \tau]) .
$$

\section{A two-state-variable model}

Consider the following example, where $d=2$ and $\phi(t, T)$ has constant norm $z$ and turns at constant angular velocity $\omega$ :

$$
\phi(t, t+\tau)=z C(\omega, \tau)
$$

where

$$
C(\omega, \tau)=\left(\begin{array}{c}
\cos [\omega \tau] \\
\sin [\omega \tau]
\end{array}\right)
$$

We prove below that this choice for $\phi(t, T)$ leads to processes for the short rate $r(t)$ and its drift $x(t)$ of the form (18-19), where $\zeta(t)=z$ and

$$
y(t)=Y(r(t), z)=\omega^{2}(\mu-r(t)) .
$$


This is a model of the yield curve in which the two state variables $r(t)$ and $x(t)$ form a Markovian vector. The short rate is stationary and has unconditional mean equal to $\mu$, while its drift $x(t)$ is also stationary and its unconditional drift is zero. Volatilities are constant, and therefore the model is Gaussian. Finally, the market price of risk is given by

$$
\lambda(t)=q \phi(t, t)=\left(\begin{array}{c}
q z \\
0
\end{array}\right)
$$

With linear drifts, constant volatilities, and a constant price of risk, the model belongs to the exponential-affine class introduced by Duffie and Kan [1993]. Its solution for forward rates is

$$
f(t, t+\tau)=F_{q}(r(t), x(t), \tau) .
$$

Using the methods described in Fisher and Gilles (1996), it is possible to verify that the conditional expectation of the short rate is

$$
E_{t}[r(t+\tau)]=F_{1}(r(t), x(t), \tau) .
$$

Clearly, this forecast is independent of the value of $q$, as it must be given that the process for $(r(t), x(t))$ is independent of $q$. But the value of $q$ affects the market price of risk, and therefore the shape of the yield curve given in (21). From these equations, it is clear that the term premia are given by

$$
\psi(t, t+\tau)=f(t, t+\tau)-E_{t}[r(t+\tau)]=(q-1) z^{2}(1-\cos [\omega(\tau)]),
$$

which agrees with the term premia under the $q$-EH as given in equation (17). In particular, under the unbiased expectations hypothesis $(q=1)$ all term premia vanish.

\section{The example is the canonical Gaussian model}

This example has a two-dimensional Markovian state vector with deterministic volatility - the Gaussian case. In Gaussian models, term premia are nonrandom functions of maturity. It would be interesting to find out how to construct non-Gaussian models of the $q-\mathrm{EH}$, because in such models term premia change randomly. We construct such examples by generalizing the canonical example.

Before turning to the issue of non-Gaussian models, however, we prove two results about the canonical example, which clearly show that it is the place whence to generalize. First, we show that there exists no one-state variable model of the $q-\mathrm{EH}$, Gaussian or not. This is simply because, under the $q-\mathrm{EH}$, the univariate process for the short rate cannot be Markovian (all proofs appear in Appendix B). 
Proposition 1 If the $q-E H$ holds and the short rate $r(t)$ is not deterministic, then its univariate process is not Markovian.

Second, we show that any Gaussian model of the $q-\mathrm{EH}$ with a two-dimensional Markovian state vector amounts to a renormalization of the canonical example.

Proposition 2 Suppose that the $q-E H$ holds in a model with two Markovian state variables in which bond prices have deterministic volatilities. Then there exist constant scalars $\mu, \omega$, and $z$ such that, perhaps after changing the basis for the vector of Brownian motions (thus affecting the representation of the processes, but not the form of the yield curve):

- $\phi(t, T)$ has the form shown in (20);

- the processes for the short rate and its drift have the form shown in equations (18) and (19), with $\zeta(t)=z$ and $y(t)=Y(r(t), z)$;

- and the initial yield curve has the form

$$
f(0, \tau)=F_{q}(r(0), x(0), \tau)
$$

Proposition 2 asserts that in a Gaussian and Markovian economy (with two state variables), the $q$-EH implies that $\phi(t, T)$ keeps turning around the circle at constant angular velocity, $\omega$. It also specifies $x(t)$, the drift of $r(t)$, as linearly independent of $r(t)$, and $y(t)$, the drift of $x(t)$, as a translation of $r(t)$ with coefficient $-\omega^{2}$, and independent of the value of $x(t)$ itself. The short rate is stationary with unconditional mean equal to $\mu$, while its drift is also stationary with unconditional mean equal to zero.

Because the model is Markovian, the initial time has no particular significance, and equation (23) for the "initial" yield curve delivers the form of the generic yield curve, which indeed agrees with the solution that follows from solving a Duffie-Kan model, as we did to get (21). But while the Duffie-Kan method requires solving a simultaneous system of three Ricatti diferential equations, we obtained the initial yield curve in the proof of Proposition 2 by solving a single second-order differential equation.

Clearly, yield curves can be flat; in fact the yield curve is flat if and only if $r(t)=\mu+(q-1) z^{2}$ and $x(t)=0$ :

$$
F_{q}\left(\mu+(q-1) z^{2}, 0, \tau\right)=\mu+(q-1) z^{2} .
$$

There is always an ergodic yield curve that is obtained by setting $r(t)$ and $x(t)$ at their respective unconditional means, $\mu$ and 0 :

$$
F_{q}(\mu, 0, \tau)=\mu+(q-1) z^{2}(1-\cos [\omega \tau]) .
$$


The ergodic yield curve is thus the same as the flat yield curve under the U-EH $(q=1)$, but in other cases it is a sine wave.

\section{Non-Gaussian models}

We now turn to the non-Gaussian case. The simplest way to generalize the canonical Gaussian example is to suppose that $\phi(t, t)$-which is porportional to the market price of risk $\lambda(t)$ - is an Ito process. To do this without increasing the number of state variables, replace equation (20) by $\phi(t, t+\tau)=\zeta(t) C(\omega, \tau)$ where $\zeta(t)$ is some function of $r(t)$ and $x(t)$. There result non-Gaussian two-state variable Markov models of the yield curve in which the $q-\mathrm{EH}$ holds. Although this strategy works well and delivers closed-form expressions for bond prices, checking that the $q$-hypothesis holds may not be easy in practice, because we do not have closed-form expressions for the conditional forecasts of the state variables. For this reason, we also introduce

a three-state variable non-Gaussian model in which we know how to compute both bond prices and conditional forecasts.

Proposition 3 Let $\phi(t, t+\tau)=\zeta(t) C(\omega, \tau)$, where $C(\omega, \tau)$ is as in (20) and $\zeta(t)=\zeta(r(t), x(t))$, for any function $\zeta(\cdot, \cdot)$ (with the restriction that the implied stochastic processes for $r(t)$ and $x(t)$ have a solution). Suppose also that $\zeta^{2}(t)$ has an unconditional mean $z^{2}$. Pick a constant $\mu$ and initial conditions $r(0)$ and $x(0)$, and choose the following initial yield curve

$$
f(0, \tau)=F_{q}(r(0), x(0), \tau) .
$$

Then:

- the resulting yield curve model is Markovian with a two-dimensional state vector $(r(t), x(t))$, as well as non-Gaussian if $\zeta(t)$ is random, and it satisfies the $q$ expectations hypothesis;

- the processes for the short rate and its drift have the form shown in equations (18) and (19), with $y(t)=Y(r(t), \zeta(t))$;

- at any time $t$, the yield curve is

$$
f(t, t+\tau)=F_{q}(r(t), x(t), \tau) .
$$

We see that bond prices are independent of $\zeta(t)$ and depend on the other two state variables $r(t)$ and $x(t)$ exactly as they do in the corresponding Gaussian model. The only difference between the Gaussian and the non-Gaussian models is the distribution of these state variables; therefore yield curves of a given shape do not occur with the same frequency in both models. 
In the non-Gaussian model, the drift of $x(t)$ depends on $\zeta^{2}(t)$ (except when $q=1$ ), which complicates the task of making conditional forecasts. If $\zeta^{2}(t)$ were a linear function of $x(t)$ and $r(t)$, then the model would be in the exponential-affine class, and we would know how to compute conditional forecasts. Unfortunately, in our two-factor model there is no guarantee that either the interest rate or its drift can stay positive (in fact, the mean of $x(t)$ equals zero), and no linear combination of these variables is guaranteed to stay positive. Therefore, $\zeta^{2}(t)$ cannot be a linear function of $(r(t), x(t))$. We can get around this problem by adding a third, independent state variable, however. The following three-state-variable model belongs to the exponential-affine class.

Proposition 4 Set $d=3$. Let $\phi(t, t+\tau)=\zeta(t) C^{*}(\omega, \tau)$, where $C^{*}$ extends the function $C$ given in (20) by adding a third component which identically equals zero; let the process for $\zeta^{2}(t)$ satisfy

$$
d \zeta^{2}(t)=k\left(z^{2}-\zeta^{2}(t)\right) d t+\zeta(t) d W_{3}(t)
$$

and let $f(0, \tau)=F_{q}(r(0), x(0), \tau)$, so that the initial yield curve is as in Proposition 3. Then:

- the resulting yield curve model is Markovian with a three-dimensional state vector $\left(r(t), x(t), \zeta^{2}(t)\right)$, as well as non-Gaussian, and it satisfies the q-expectations hypothesis;

- the processes for the short rate and its drift have the form shown in equations (18) and (19), with $y(t)=Y(r(t), \zeta(t))$;

- at any time $t$, the yield curve is

$$
f(t, t+\tau)=F_{q}(r(t), x(t), \tau)
$$

The bond price formula in the three-state-variable model is identical to that in the two-state-variable model. The only difference is that, because the former model belongs to the exponential-affine class, it is possible to obtain closed-form solutions for the conditional forecasts of all state variables (as well as their conditional variances). The state variable of most interest, of course, is the short rate itself, for which we have:

$$
\begin{aligned}
E_{t}[r(t+\tau)]= & F_{1}(r(t), x(t), \tau)+ \\
& \frac{(1-q) \omega^{2}}{k^{2}+\omega^{2}}\left(\zeta^{2}(t)-z^{2}\right)\left(e^{-k \tau}-\cos [\omega \tau]+\frac{k \sin [\omega \tau]}{\omega}\right) .
\end{aligned}
$$

It can then be verified that the forward premium is

$$
\psi(t, t+\tau)=f(t, t+\tau)-E_{t}[r(t+\tau)]
$$




$$
=(q-1)\left(z^{2}(1-\cos [\omega \tau])-\frac{\omega^{2}\left(\zeta^{2}(t)-z^{2}\right)}{k^{2}+\omega^{2}}\left(e^{-k \tau}-\cos [\omega \tau]+\frac{k \sin [\omega \tau]}{\omega}\right)\right) .
$$

It can be further verified that, because

$$
E_{t}\left[\zeta(t+s)^{2}\right]=z^{2}+e^{-k s}\left(\zeta(t)^{2}-z^{2}\right)
$$

the above expression for the term premium agrees with (17), which in the present case reduces to

$$
\psi(t, t+\tau)=(q-1) \omega \int_{s=0}^{\tau} E_{t}\left[\zeta(t+s)^{2}\right] \sin [\omega(\tau-s)] d s .
$$

\section{Concluding remarks}

We have shown that the expectations hypothesis is compatible with general equilibrium even in Markovian settings. The models we have been able to construct, however, will not help to rehabilitate the expectations hypothesis. Rather, they show how implausible the hypothesis is. The main reason all expectations hypotheses are implausible in Markovian settings is that they share the same process for the short rate, which implies that the forecast of the short rate path is a sine wave with nondampening amplitude. Of course, forward rates behave in the same way (they are equal to the short rate forecast under the unbiased expectations hypothesis).

In a non-Markovian setting, yield curves and forecasts of the path of the short rate can look more reasonable. Note that in his example, McCulloch did not exhibit a yield curve. In fact, McCulloch's example is compatible with any initial yield curve. ${ }^{14}$ The expectations hypothesis imposes restrictions only on the dynamics of the yield curve. Given the initial yield curve and its dynamics, it is in principle possible to reconstruct future yield curves for any path of the set of Brownian motions. But because there is no finite set of variables that summarizes the state of the economy, we cannot say what a typical yield curve looks like.

At first blush, it may seem that the Markovian models have the potential to represent the cyclical behavior of interest rates prior to the existence of the Federal Reserve. Unfortunately, the models cannot be made to reasonably approximate that sort of cyclical behavior. The problem is that the pre-Fed cycle occurs in absolute time, while the cycles in the Markovian models occur in relative time. In other words, there is no way to make summer (for example) be a high (or low) rate season on average.

As a final observation, we suspect that no equilibrium model of the expectations hypothesis, Markovian or non-Markovian, can guarantee the non-negativity of the

\footnotetext{
${ }^{14}$ Initial yield curves in our examples are determined only by the condition that the model is Markovian, as the proofs of the propositions make clear.
} 
short rate. This is certainly true in McCulloch's example and all of our examples. Such a feature makes the expectations hypothesis a poor benchmark for nominal rates. The reason for the inability to keep the short rate positive is simple. If the short rate is to stay positive, its volatility must be small enough and its drift must be positive whenever its level is close to zero. But in all our examples, the drift of the short rate is independent of the short rate itself, and therefore will not always point in the right direction when the rate is small. 


\section{Appendices}

\section{A Absence-of-arbitrage and general equilibrium}

In this Appendix we derive the HJM absence-of-arbitrage restriction and we show the relationship to general equilibrium models. Much of the material can be found in Duffie (1996).

\section{Absence-of-arbitrage}

There are several equivalent ways to impose the no-arbitrage condition on the process for bond prices. The most direct, perhaps, is to postulate the existence of a strictly positive process $m(t)$, which obeys

$$
\frac{d m(t)}{m(t)}=\mu_{m}(t) d t+\sigma_{m}(t)^{\top} d W(t)
$$

such that the value at time $t, V(t)$, of any payoff $D(T)$ at time $T$ obeys

$$
V(t) m(t)=E_{t}[D(T) m(T)]
$$

as a result, the deflated value $V(t) m(t)$ is a martingale. Following Duffie (1996), we call $m(t)$ the state-price deflator.

Define the conditional expectation at time $t$ of the state-price deflator at time $T$ : $z(t, T):=E_{t}[m(T)]$; then $z(t, T)$ is a strictly positive martingale whose process can be written as follows:

$$
\frac{d z(t, T)}{z(t, T)}=\sigma_{z}(t, T)^{\top} d W(t)
$$

which, by Ito's lemma, implies

$$
d \log [z(t, T)]=-\frac{1}{2}\left\|\sigma_{z}(t, T)\right\|^{2} d t+\sigma_{z}(t, T)^{\top} d W(t) .
$$

In view of (A.2) specialized to the case $D(T)=1$, the term structure of interest rates can be written as

$$
P(t, T)=\frac{z(t, T)}{m(t)}
$$

We now relate the process for the state-price deflator to the short rate and the so-called market price of risk. To do this, define the money market account $\beta(t)$ as

the value of the particular asset that consists of the accumulation of one unit of the 
numeraire continuously reinvested at the short rate:

$$
\beta(t):=\exp \left(\int_{s=0}^{t} r(s) d s\right)
$$

which implies that the process for $\beta(t)$ is

$$
\frac{d \beta(t)}{\beta(t)}=r(t) d t
$$

In view of (A.1) and (A.6), Ito's lemma implies that the proportional drift of $\beta(t) m(t)$ is $r(t)+\mu_{m}(t)$. Since $\beta(t)$ is the value of an asset, however, $\beta(t) m(t)$ must be a martingale, and therefore its drift must equal zero. It immediately follows that

$$
\mu_{m}(t)=-r(t)
$$

Similarly, in view of (9) and (A.1), Ito's lemma implies that the proportional drift of $m(t) P(t, T)$ is $\mu_{m}(t, T)+\mu_{P}(t)+\sigma_{P}(t, T)^{\top} \sigma_{m}(t)$. Since the deflated bond price $P(t, T) m(t)=E_{t}[m(T)]$ is a martingale, we must have

$$
\mu_{P}(t, T)=r(t)+\sigma_{P}(t, T)^{\top} \lambda(t)
$$

where $\lambda(t)$, which we call the market price of risk, is defined as $\lambda(t):=-\sigma_{m}(t)$. Therefore we can always write the process for the state-price deflator as

$$
\frac{d m(t)}{m(t)}=-r(t) d t-\lambda(t)^{\top} d W(t)
$$

which, by Ito's lemma, implies

$$
d \log [m(t)]=-\left(r(t)+\frac{1}{2}\|\lambda(t)\|^{2}\right) d t-\lambda(t)^{\top} d W(t)
$$

Next, applying Ito's lemma to (A.5) produces the following relationship:

$$
\sigma_{P}(t, T)=\sigma_{z}(t, T)+\lambda(t)
$$

Since $z(t, t)=m(t)$, we have $\sigma_{z}(t, t)=-\lambda(t)$ and as a result $\sigma_{P}(t, t)=0$.

We now turn to the no-arbitrage condition in the HJM framework. This condition states that in (3), the drift $\mu_{f}(t, T)$ cannot be chosen independently of $\lambda(t)$ and $\sigma_{f}(t, T)$. To see this, note that equations (1) and (A.5) imply

$$
f(t, T)=-\frac{\partial}{\partial T} \log [z(t, T)]
$$


from which it follows that

$$
d f(t, T)=-d\left(\frac{\partial}{\partial T} \log [z(t, T)]\right) .
$$

In view of the process for $\log [z(t, T)]$ given in (A.4), we have

$$
\sigma_{f}(t, T)=\sigma_{z}^{\prime}(t, T)
$$

and

$$
\mu_{f}(t, T)=\frac{1}{2} \frac{\partial}{\partial T}\left\|\sigma_{z}(t, T)\right\|^{2}=\sigma_{z}^{\prime}(t, T)^{\top} \sigma_{z}(t, T) .
$$

In the spirit of HJM, considering $\sigma_{f}$ (rather than $\sigma_{z}$ ) as a primitive, then, equations (A.9), (A.10) and (A.11) imply (5).

\section{Relationship to general equilibrium}

Any model of the term structure that is free of arbitrage can be interpreted as a general-equilibrium model. We now give our model such an interpretation. Assume an exchange economy with a representative consumer who maximizes the expected discounted sum of instantaneous utility with constant discount factor $\rho$,

$$
E_{t}\left[\int_{t}^{\infty} e^{-\rho s} u(c(s)) d s\right]
$$

Let the instantaneous utility be of the constant relative risk aversion class, with risk aversion parameter $\eta$ :

$$
u(c):= \begin{cases}\frac{c^{1-\eta}-1}{1-\eta} & \text { for } 0 \leq \eta<1 \text { or } 1<\eta \\ \log (c) & \text { for } \eta=1,\end{cases}
$$

so marginal utility at time $t$ is

$$
m(t)=e^{-\rho t} c(t)^{-\eta}
$$

where $c(t)$ is consumption at time $t$. In these models marginal utility is the state-price deflator. ${ }^{15}$ The process for the log of consumption can be found by solving (A.12) for $\log [c(t)]:$

$$
\begin{aligned}
d \log [c(t)] & =-\frac{1}{\eta}(d \log [m(t)]+\rho d t) \\
& =\tilde{\mu}_{c}(t) d t+\sigma_{c}(t)^{\top} d W(t)
\end{aligned}
$$

\footnotetext{
${ }^{15}$ See Duffie (1996), pp. 225-226.
} 
where, using equation (A.8),

$$
\tilde{\mu}_{c}(t)=\frac{1}{\eta}\left(r(t)+\frac{1}{2}\|\lambda(t)\|^{2}-\rho\right)
$$

and

$$
\sigma_{c}(t)=\frac{\lambda(t)}{\eta}
$$

From (13) and (A.14) we see that under the $q-\mathrm{EH}, \sigma_{c}(t)=(q / \eta) \phi(t, t)$.

Finally, note that equation (A.11) allows us to write the forward premium as

$$
\psi(t, T)=-\frac{1}{2} \int_{v=t}^{T} E_{t}\left[\frac{\partial}{\partial T}\left\|\sigma_{z}(v, T)\right\|^{2}\right] d v .
$$

When the state-price deflator is interpreted as the marginal utility of consumption, (A.15) expresses in continuous time Woodward's (1983) characterization of the term premium. In particular, we see that the forward premium is zero if the relative standard deviation (the coefficient of variation - in Woodward's terminology) of the conditional expectation of the marginal utility of consumption is independent of the horizon; i.e., if $\partial / \partial T\left\|\sigma_{z}(v, T)\right\| \equiv 0$.

\section{B Proofs of Propositions}

To prove the propositions, we need the process for the short rate under the $q-\mathrm{EH}$. In the HJM framework, the three model primitives - the initial yield curve, the market price of risk and the volatility of either forward rates - are guaranteed to deliver an arbitrage-free model of the term structure. The short rate is given by

$$
r(t)=f(t, t)=f(0, t)+\int_{s=0}^{t} \mu_{f}(s, t) d s+\int_{s=0}^{t} \sigma_{f}(s, t)^{\top} d W(s),
$$

where $\mu_{f}(t, T)$ is given by equation (5). Clearly, from (B.1), the process for the short rate obeys

$$
\begin{aligned}
d r(t)=\{ & \frac{\partial}{\partial t} f(0, t)+\mu_{f}(t, t)+\int_{s=0}^{t} \frac{\partial}{\partial t} \mu_{f}(s, t) d s \\
& \left.+\int_{s=0}^{t} \frac{\partial}{\partial t} \sigma_{f}(s, t)^{\top} d W(s)\right\} d t+\sigma_{f}(t, t)^{\top} d W(t)
\end{aligned}
$$

Note that the short rate process in equation (B.2) is not necessarily Markovian for an arbitrary $\left\{\lambda(t), \sigma_{f}(t, T)\right\}$ pair, although it can be one of the components of a Markovian state vector in an economy where the $q-\mathrm{EH}$ holds. Using (14) and imposing 
the $q$-EH condition (16), the short rate, given in (B.1), becomes

$$
r(t)=f(0, t)+(q-1) \int_{s=0}^{t} \phi^{\prime}(s, t)^{\top} \phi(s, s) d s+\int_{s=0}^{t} \phi^{\prime}(s, t)^{\top} d W(s)
$$

from which it follows that the process for the short rate obeys

$$
d r(t)=x(t) d t+\phi^{\prime}(t, t)^{\top} d W(t)
$$

where $^{16}$

$$
x(t)=f^{\prime}(0, t)+(q-1) \int_{s=0}^{t} \phi^{\prime \prime}(s, t)^{\top} \phi(s, s) d s+\int_{s=0}^{t} \phi^{\prime \prime}(s, t)^{\top} d W(s) .
$$

From this definition, the process for $x(t)$ clearly obeys

$$
d x(t)=y(t) d t+\phi^{\prime \prime}(t, t)^{\top} d W(t)
$$

where

$$
\begin{aligned}
y(t)=f^{\prime \prime} & (0, t)+(q-1) \phi^{\prime \prime}(t, t)^{\top} \phi(t, t)+ \\
& (q-1) \int_{s=0}^{t} \phi^{\prime \prime \prime}(s, t)^{\top} \phi(s, s) d s+\int_{s=0}^{t} \phi^{\prime \prime \prime}(s, t)^{\top} d W(s) .
\end{aligned}
$$

The difficulty is to guarantee that the short rate is driven by a Markov state vector. The short rate itself cannot be Markovian, except in the trivial case of no uncertainty, in view of Proposition 1.

\section{Proof of Proposition 1}

If $r(t)$ is Markovian, then (i) its drift $x(t)$ must be a function of $r(t)$, say $x(t)=$ $g(r(t))$, for some continuous function $g(\cdot)$; and its volatility must be of the form $\phi^{\prime}(t, t)=h_{2}(r(t), 0)$, where $\phi(t, t+\tau)=h(r(t), \tau)$, the function $h$ is differentiable and $h_{i}$ denotes the derivative with respect to the second variable. Fix a time $s$ and consider a disturbance in the path of $W(t)$ by $\Delta W(s)$. For simplicity, we assume $g$ to be differentiable. Then from equations (B.3) and (B.5), we see that change at time $s$ in the short rate and its drift would have been

$$
\Delta r(s)=h_{2}(r(s), 0)^{\top} \Delta W(s),
$$

and

$$
\Delta x(s)=h_{22}(r(s), 0)^{\top} \Delta W(s) .
$$

\footnotetext{
${ }^{16}$ Note that $\mu_{f}(t, t)=0$, a result that follows directly from (15).
} 
But for $\Delta W(s)$ small enough, we must also have $\Delta x(s)=g^{\prime}(r(s)) \Delta r(s)$. This equation must be satisfied for any value of $r(s)$,

$$
h_{22}(r(s), 0)=g^{\prime}(r(s)) h_{2}(r(s), 0) \quad \text { for all } s \leq t .
$$

Since $g^{\prime}$ is a scalar, this equation means that $h_{22}(r(s), 0)$ is proportional to $h_{2}(r(s), 0)$. Unless $h_{2}=h_{22}=0$, which is the deterministic case, $h_{22}=\phi^{\prime \prime}$ cannot be proportional to $h_{2}=\phi^{\prime}$ because that would drive $\phi$ outside the circle. Q. E. D.

\section{Proof of Proposition 2}

In a two-factor model, we can set $d=2$. From Proposition 1, we know that the short rate is not Markovian, and therefore its drift, $x(t)$, is not a function of $r(t)$. But in a two-state variable Markovian model, the vector $(r(t), x(t))$ is driven by a two-dimensional Markovian vector, and is Markovian itself. In other words, it is legitimate to take $r(t)$ and $x(t)$ as the two state variables. This requires that $y(t)$, the drift of $x(t)$, be a function of the state variables. Assume, then, that for some continuous function $g(\cdot, \cdot), y(t)=g(r(t), x(t))$.

We assume without loss of generality that $\phi(t, t)$ has a non-zero entry only in its first component, and we write $\phi(t, t)=(\zeta(t), 0)$, for some scalar process $\zeta(t)$ to be determined later. There is no loss of generality because, if $\phi(t, t)=0$, it has the correct form to start with, whereas if $\phi(t, t) \neq 0$, then we can redefine the basis of Brownian motions as follows: Let $B(t)=\left(B_{1}(t), B_{2}(t)\right)$ be an orthonormal set of Brownian motions such that $B_{1}(t)=\phi(t, t)^{\top} W(t) /\|\phi(t, t)\|$; the other component of $B(t)$ is chosen in the span of $W_{1}(t)$ and $W_{2}(t)$ to be orthonormal to $B_{1}(t)$. This procedure amounts to choosing a Choleski decomposition of the noise in the economy such that the noise in the state-price deflator comes first (recall that $\phi(t, t)$ and $\lambda(t)$ are co-linear, and $-\lambda(t)$ is the volatility of the state price deflator $\left.{ }^{17}\right)$. From now on, we assume that $W(t)$ was chosen to start with to coincide with the basis $B(t)$.

Before proceeding further, we re-parametrize the function $\phi$ using polar coordinates to enforce the restrictions that $\phi(t, t)=(\zeta(t), 0)$ and that $\phi(t, T)$ lies on a circle. Write

$$
\phi(t, T)=\zeta(t)\left(\begin{array}{c}
\cos [\theta(t, T)] \\
\sin [\theta(t, T)]
\end{array}\right)
$$

where $\theta(t, T)$, the angle determining the position of $\phi(t, T)$ on the circle, satisfies $\theta(t, t)=0$. Clearly, $\|\phi(t, T)\|=\|\phi(t, t)\|=|\zeta(t)|$. By differentiation, we get

$$
\phi^{\prime}(t, T)=\zeta(t) \theta^{\prime}(t, T)\left(\begin{array}{r}
-\sin [\theta(t, T)] \\
\cos [\theta(t, T)]
\end{array}\right)
$$

\footnotetext{
${ }^{17}$ See Appendix A.
} 


$$
\phi^{\prime \prime}(t, t)=\zeta(t)\left\{\theta^{\prime \prime}(t, T)\left(\begin{array}{r}
-\sin [\theta(t, T)] \\
\cos [\theta(t, T)]
\end{array}\right)-\theta^{\prime}(t, T)^{2}\left(\begin{array}{c}
\cos [\theta(t, T)] \\
\sin [\theta(t, T)]
\end{array}\right)\right\}
$$

and

$$
\begin{aligned}
\phi^{\prime \prime \prime}(t, T)=\zeta(t)\left\{\theta^{\prime \prime \prime}(t, T)\left(\begin{array}{r}
-\sin [\theta(t, T)] \\
\cos [\theta(t, T)]
\end{array}\right)-3 \theta^{\prime \prime}(t, T) \theta^{\prime}(t, T)\left(\begin{array}{r}
\cos [\theta(t, T)] \\
\sin [\theta(t, T)]
\end{array}\right)\right. \\
\left.-\theta^{\prime}(t, T)^{3}\left(\begin{array}{r}
-\sin [\theta(t, T)] \\
\cos [\theta(t, T)]
\end{array}\right)\right\}
\end{aligned}
$$

We now show that $\phi(t, T)$ is deterministic. Because bond prices have non-random volatilities by assumption, $\phi^{\prime}(t, T)=\sigma_{f}(t, T)$ must be deterministic. From the form of $\phi^{\prime}$ in (B.8), it is clear that if $\theta^{\prime} \neq 0$ (ruling out the trivial case where bond prices are deterministic), $\zeta(t)$ must be deterministic in order for $\phi^{\prime}(t, T)$ to be deterministic, so that $\phi(t, T)$ is itself deterministic. In other words, the $q-\mathrm{EH}$ imposes the restriction that if bond prices have constant variance, then the price of risk $\lambda(t)$ is constant.

We now turn to the implications of the $q-\mathrm{EH}$ for the form of $g$ and $\phi(t, T)$. For simplicity, assume that $g$ is differentiable (with this assumption, we find that it is in fact linear), and denote by $g_{1}$ and $g_{2}$ its partial derivatives with respect to $r(t)$ and $x(t)$. We use the variation method used in the previous proof: fix a time $t$ and disturb the path of $\{W(s) \mid s>0\}$ at some point in the past $\tau<t$. Since $\phi(t, T)$ is deterministic, it is unaffected by this change; as a result, the short rate $r(t)$, its drift $x(t)$, and $y(t)$, the drift of $x(t)$, change by the amounts

$$
\begin{aligned}
\Delta r(t) & =\phi^{\prime}(\tau, t) \Delta W(\tau), \\
\Delta x(t) & =\phi^{\prime \prime}(\tau, t) \Delta W(\tau),
\end{aligned}
$$

and

$$
\Delta y(t)=\phi^{\prime \prime \prime}(\tau, t) \Delta W(\tau)
$$

But we must have also (for infinitesimal changes)

$$
\Delta y(t)=g_{1}(r(t), x(t)) \Delta r(t)+g_{2}(r(t), x(t)) \Delta x(t)
$$

These equations must hold for any value of $(r(t), x(t))$, and any $\tau$. We conclude that $g_{1}$ and $g_{2}$ must be constant, so that, for some scalars $a, b$ and $c$, we can write

$$
y(t)=g(r(t), x(t))=a+b r(t)+c x(t) .
$$

Moreover, given the forms of $\Delta r(t), \Delta x(t)$ and $\Delta y(t)$, equation (B.12) holds if and only if

$$
\phi^{\prime \prime \prime}(t, T)=b \phi^{\prime}(t, T)+c \phi^{\prime \prime}(t, T)
$$


Substituting (B.8-B.10) into equation (B.13) allows us to conclude that that restriction will be violated unless, for some constant $\omega$,

$$
\theta^{\prime}(t, T)=\omega ; \quad b=-\omega^{2} ; \quad \text { and } \quad c=0 .
$$

Since $\theta(t, t)=0, \theta^{\prime}=\omega$, a constant, implies $\theta(t, T)=\omega(T-t)$. Since $\zeta(t)$ is not stochastic, it must be constant (otherwise, absolute time would enter as an independent argument of $g$, so that, strictly speaking, $(r(t), x(t))$ would not be Markovian); let $z$ denote the constant value of $\zeta(t)$. This establishes the first two parts of the proposition, which have to do with the form of $\phi$ and, as a result, the processes for $r(t)$ and its drift, $x(t)$.

The constant $a$ in (B.12) is arbitrary, so that the drift of $x(t)$ is $y(t)=$ $g(r(t), x(t))=a-\omega^{2} r(t)$; but we let $a=\mu \omega^{2}$, and choose to parameterize $g$ in terms of $\mu$ and $\omega$, rather than $a$ and $\omega$.

To finish the proof, we need to find the form of the initial yield curve. Given the form of $\phi(t, T)$, equations (B.3) and (B.7) imply that

$$
y(t)=f^{\prime \prime}(0, t)+\omega^{2}(f(0, t)-r(t)),
$$

from which we conclude that

$$
a=\mu \omega^{2}=f^{\prime \prime}(0, t)-(q-1) \omega^{2} \zeta^{2}+\omega^{2} f(0, t) .
$$

This is a second order ordinary differential equation, a solution of which requires two boundary conditions. These conditions come from the initial conditions for the state of the system, $r(0)$ and $x(0)$. Clearly, $f(0, t)=r(0)$ - this also follows mechanically from equation (B.3) - and from equation (B.5), we see that $f^{\prime}(0, t)=x(0)$. The unique solution to (B.14) subject to these initial conditions is (23). Q. E. D.

\section{Proof of Proposition 3}

We start by verifying that the second statement, about the processes of $r(t)$ and $x(t)$. The drift of $r(t)$ is $x(t)$ by definition. Given the assumed form of $\phi(t, T)$, the volatilities of $r(t)$ and $x(t)$ are $(0, \omega \zeta(t))$ and $\left(-\omega^{2} \zeta(t), 0\right)$, as stated. The drift of $x(t), y(t)$, can be calculated from (B.7). Given (B.3) and the form of $\phi(t, T)$, we see that $y(t)=f^{\prime \prime}(0, t)-\omega^{2}\left(r(t)-f(0, t)+(q-1) \zeta^{2}(t)\right)$. The stated result follows from the assumed form of the initial yield curve, which implies that $f^{\prime \prime}(0, t)+\omega^{2} f(0, t)=$ $\omega^{2}\left(\mu+(q-1) z^{2}\right)$.

We now turn to the first and third statements. The fact that the model satisfies the $q$-expectations hypothesis is built into the form of $\phi(t, T)$. The form of the processes for the short rate and its drift clearly show that $(r(t), x(t))$ is a Markovian vector, since $\zeta(t)$ is assumed to be a deterministic function of this vector. If the model 
of the yield curve is Markovian, then given the value of the state vector, it should not independently matter what value the time index $t$ has. In particular, equation (B.7) must still hold for any reference date $t$ replacing date 0 (and $t+\tau$ replacing $t$ ). This implies that for any $t, f^{\prime \prime}(t, t+\tau)+\omega^{2} f(t, t+\tau)=\omega^{2}\left(\mu+(q-1) z^{2}\right)$. The only solution of this differential equation (where $t$ is fixed and the variable is $\tau$ ) satisfying the boundary conditions $f(t, t)=r(t)$ and $f^{\prime}(t, t)=x(t)$ is

$$
f(t, t+\tau)=\left(\mu+(q-1) z^{2}\right)(1-\cos [\omega \tau])+r(t) \cos [\omega \tau]+\frac{x(t) \sin [\omega \tau]}{\omega} .
$$

Q. E. D.

\section{Proof of Proposition 4}

The proof is identical to that of the previous proposition. Q. E. D. 


\section{References}

[1] Campbell, J., 1986, A defense of traditional hypotheses about the term structure of interest rates. Journal of Finance 41, 183-193.

[2] Campbell, J., and R. Shiller, 1991, Yield spreads and interest rate movements: A bird's eye view. Review of Economic Studies 58, 495-514.

[3] Cox, J., J. Ingersoll, and S. Ross, 1981, A reexamination of traditional hypotheses about the term structure of interest rates, Journal of Finance 36, 321-346.

[4] Duffie, D., 1996, Dynamic Asset Pricing Theory (Princeton University Press, Princeton, New Jersey, 2nd ed.).

[5] Duffie, D. and R. Kan, 1993, A yield-factor model of interest rates (Working paper, Stanford University).

[6] Frachot, A. and J.-P. Lesne, 1994, Expectations hypotheses and stochastic volatilities (Working paper, Banque de France).

[7] Fisher, M. and C. Gilles, 1996, Term premia in an exponential-affine economy (Working paper, Federal Reserve Board).

[8] Heath, D., R. Jarrow, and A. Morton, 1992, Bond pricing and the term structure of interest rates: a new methodology. Econometrica 60, 77-105.

[9] Hull, J., 1993, Options, futures, and other derivative securities (Prentice Hall, New Jersey, 2nd. ed.).

[10] McCulloch, J. H., 1993, A reexamination of traditional hypotheses about the term structure of interest rates: A comment Journal of Finance 48, 779-789.

[11] Woodward, S., 1983, The liquidity premium and the solidity premium American Economic Review 73, 348-361. 